\title{
Review: Polymers for Mucoadhesive Drug Delivery Systems
}

\author{
Sunita Devi ${ }^{1 *}$, Meenakshi Bhatia ${ }^{1}$, Samiksha Grewal ${ }^{1}$ \\ 1 Guru Jambheshwar University of science and technology, pharmaceutical sciences, Hisar, India
}

\begin{abstract}
In bio adhesive drug delivery, the word bio adhesion is used to define the connection between polymers (synthetic or natural) and gastro-intestinal mucosa or any soft tissue of the body. In cases when the bond is designed with the mucosa, the term mucoadhesion may be used synonymously with bio adhesion. For delivered the mucoadhesive drug by oral route following locations are used:-Oral cavities (lips, tongue, cheek or buccal, soft palate, hard palate and floor of the mouth. Importance of Antioxidant activity, it increase the dwelling time of the dosage time at the absorption site and it improves absorption and the therapeutic ability of the drug due to increase the residence time. Natural polysaccharides have an extensive variety of applications as a dietary supplement and pharmaceutical excipients with good biocompatibility, low cost, and easy accessibility. Polysaccharides are obtained from various sources like plants, marine, and animals.
\end{abstract}

Keywords: Polymers, Mucoadhesive drug delivery system, Antioxidant activity, Flaxseed mucilage, and Grafted Polysaccharides.

\section{INTRODUCTION}

Polysaccharides are biopolymers made up from different and lengthy chains of monosaccharides units which are joined together by a glycosidic link and on hydrolysis reactions yields oligo and monosaccharides which are known as polysaccharides. Polysaccharides are related to carbohydrates and 99\% are founds in plants ${ }^{1}$. Polysaccharides are often quite homogenous, containing slight modifications of repeating units. Frequently, polysaccharides are not in pure form.

\footnotetext{
*Corresponding author: Sunita Devi, e-mail: sunitamechu1504@gmail.com ORCIDs

Sunita Devi: 0000-0002-1660-8550

Meenakshi Bhatia: 0000-0003-4568-7582

Samiksha grewa: 0000-0002-9548-7477

Drug Delivery research laboratory department of pharmaceutical sciences, Guru Jambheshwar University of science and technology

(Received 26 April 2020, accepted 10 September 2020)
} 
They are associated with other polysaccharides, polyphenolics or proteins by covalent or non-covalent bonds. The structure of polysaccharides are frequently linear but may contain various degree of branching ${ }^{2}$.

Polysaccharides are of mainly two types:

(A) Homo Polysaccharides: They are those polysaccharides which on hydrolysis yield a single type of monosaccharides. E.g., Cellulose, Dextrin, Inulin, Starch, Glycogen, etc.

(B) Hetero Polysaccharides: When polysaccharides are composed of different types of sugars are hetero polysaccharides. E.g., Proteoglycans, Hyaluronic acid, etc.

Natural polysaccharides have an extensive variety of applications as a dietary supplement and pharmaceutical excipients with good biocompatibility, low cost, and easy accessibility. Polysaccharides are obtained from various sources like plants, marine, and animals. Polysaccharides containing various polymers such as gums and mucilage for various uses in pharmaceutical formulations ${ }^{3,4}$. When natural polysaccharides are compared with synthetic polymers, natural polysaccharides are nontoxic, economic and biodegradable. Many polysaccharides such as chitosan, xanthan gum, sodium alginate, etc. are used in combination or either alone with their customized and indigenous form to evaluated controlled release of drugs, water treatment, improved oil recovery and flocculation of suspension ${ }^{5}$. Table 1. Shows the naturally occurring polysaccharides obtained from various sources:

Table 1. List of various polysaccharides and their sources

\begin{tabular}{|c|c|c|}
\hline S.No. & Sources & Polysaccharides \\
\hline $\mathbf{1 .}$ & Plants & Starch, Pectin, Guar gum, Flaxseed gum, Pectin, inulin, Albizia gum \\
\hline $\mathbf{2 .}$ & Animals & Chitosan, Hyaluronic acid, Chitin, Chondroitin sulfate \\
\hline $\mathbf{3 .}$ & Seaweed & Alginates, Carrageenan, Agar \\
\hline $\mathbf{4 .}$ & Fungal & Scleroglucan \\
\hline $\mathbf{5 .}$ & Microbial & Xanthan gum, Gellan gum, Dextran, Glycan, Pullulan \\
\hline $\mathbf{6 .}$ & Tree exudates & Tragacanth, Gum acacia, Ghatti gum \\
\hline
\end{tabular}

Application of polysaccharides:

(A) Antitumor activity: Polysaccharides indicated important anti-tumor activity beside A375 and BGC823 cells and with vascular smooth muscles, cells show low cytotoxicity. Pectin a complex ${ }^{6}$ polysaccharide, and a significant source of dietary fiber, it shows inhibitory action for several cancer lines. Polysaccharides anticancer activity is affected by the form of molecules, size of molecules, solubility in water and degree of branching. If water solubility of polysaccharide is higher and greater molecular weight then it shows greater antitumor activity. 
(B) Immunoregulatory activity: Plant-derived polysaccharides have the best immunemodulatory constituents and have been displayed to be clinically therapeutic. Immune functions of macrophages, lymphocytes and natural killer cells directly active by plant polysaccharides.

(C) Antidiabetes activity: The polysaccharides which are obtained since natural resources (higher plants, algae, and mushrooms entirely have significant potential for the management of diabetes and also provide an annoying source for upcoming innovation and developed a new and unique compounds of therapeutic worth. Astragali Radix and Rahmanniae Radix exist two new compounds used for diabetes mellitus in China.

(D) Anticoagulant activity: After sulfate modification, anticoagulant activity of polysaccharides similar action as heparin but no anti-platelet activity and avoid the side effect which induced by heparin. Sulfated citrus pectin and sulfated glycol glucuronomannan have revealed antithrombotic and anticoagulant activity. Sulfated polysaccharides found in aquatic invertebrates and aquatic algae with anticoagulant activity.

(E) Antiviral activity: Astragalus polysaccharide displays antiviral activity beside infectious bursal disease viruses and also reduces mortality and morbidity of the IBD (Infectious bursal disease) virus-infected chicks. Polysaccharides existing in algae and their abstracts (alginate, fucoidan, and laminarin) can inhibit bacteriological as well as viral infections.

(F) Antioxidant activity: Natural polysaccharides is an extremely favorable source of anti-oxidants, exclusively those which have been capability to more unrestricted radicals, so it has definite antioxidant and antiaging activity. Algal polysaccharides play a significant part as free radical scavengers in-vitro for the anticipation of oxidative impairment inactive organisms.

\section{Polymers used for mucoadhesive drug delivery system ${ }^{7}$}

Before using any polymer for any mucoadhesive formulation, polymers have been examined to ensure if they fulfill all the requirements (high number of hydrogen bonding, swelling properties, suitable wetting and chain flexibility of polymer) to ensure diffusion of mucoadhesive polymer to the mucous membrane network. Hydration is an also important requirement for mucoadhesive polymer to create sufficient macromolecular mesh and release polymeric chains to increase elucidation between mucin and polymer. The use of mucoadhesive polymers increase day by day to modified the release of formulation dosage form and also increased their use for buccal and gastro retentive drug delivery system. The design of some bilayer polymeric mixture can provide new material 
for biomedical application and increase adhesion time, prolonged dwelling time and greater surface contact with the mucosa.

A new advancement (Polymer blending) that can combine the advantage of many different polymers and usually faster and cheaper development as compared to new monomers and new polymerization routes. Because of this reason, especially thermo-responsive and mucoadhesive polymers are used.

\section{Characteristics of ideal polymers}

- Non-irritant to mucosal membrane

- Optimum molecular weight

- Good spreadability, wetting, swelling, and biodegradable properties.

Mucoadhesive polymers in drug delivery: Table 2 shows various types of polymers which are used in mucoadhesive drug delivery system ${ }^{8,9}$.

Table 2. List of various polymers used in mucoadhesive drug delivery system

\begin{tabular}{|c|c|c|c|}
\hline Source Dependent Polymers & Aqueous solubility & Charge & $\begin{array}{c}\text { Potential Bioadhesive } \\
\text { Force }\end{array}$ \\
\hline $\begin{array}{c}\text { Natural polymers } \\
\text { E.g., Agarose, gelatin, chitosan } \\
\text { Various gums } \\
\text { (guar, xanthan, pectin, and } \\
\text { sodium alginate) } \\
\text { Synthetic polymers } \\
\text { E.g., } \\
\text { Cellulose derivatives: } \\
\text { CMC, Thiolated CMC, sodium } \\
\text { CMC, HPMC, HPC, methyl } \\
\text { hydroxyethyl cellulose. } \\
\text { Poly(acrylic acid)- based } \\
\text { polymers } \\
\text { E.g., Polyacrylates, PAA, PC, } \\
\text { Poly(methyl vinyl ether-coethyl } \\
\text { hexyl acrylates), polyoxyethylene, } \\
\text { PVP, PVA, thiolated polymers }\end{array}$ & $\begin{array}{c}\text { Water-soluble } \\
\text { E.g., HEC, HPC, sodium } \\
\text { CMC, Sodium alginate. } \\
\text { HPMC flax seed } \\
\text { water-insoluble } \\
\text { chitosan (soluble in } \\
\text { dilute aqueous acids), } \\
\text { EC, PC }\end{array}$ & $\begin{array}{l}\text { Cationic charge } \\
\text { E.g., Amino dextran, } \\
\text { Chitosan trimethylated } \\
\text { chitosan, dimethyl } \\
\text { aminoethyl- dextran } \\
\text { Anionic charge } \\
\text { E.g., chitosan, EDTA, } \\
\text { CP, CMC, Pectin, PAA, } \\
\text { PC, sodium alginate, } \\
\text { Sodium CMC, Xanthan } \\
\text { gum }\end{array}$ & $\begin{array}{c}\text { Covalent bonded } \\
\text { polymers } \\
\text { E.g., hydroxyethyl } \\
\text { starch, HPC, PVA, PVP } \\
\text { Hydrogen bond } \\
\text { E.g., Acrylates, poly } \\
\text { (methacrylic acid), CP, } \\
\text { PC, PVA } \\
\text { Electrostatic } \\
\text { interactions } \\
\text { E.g., Chitosan }\end{array}$ \\
\hline
\end{tabular}

(A) Polyacrylates: There polymers are present in a broad variety of molecular weights, definitely modified systems of gel, safe besides non-toxic for oral uses. Polycarbophil and carbomers (PAA derivatives) are most preferred used as mucoadhesive polymer. Carbopol used as a polymer in oral preparation, suspension, and tablets. Polycarbophil is water-insoluble polymer but has a high swelling property that allowing high levels of enlargement within the mucus layer. The mucoadhesive properties of PAA increased by its conjugation with cysteine $^{10}$. Sodium polyacrylate, also known as waterlock, is a sodium salt of polyacrylic acid with the chemical formula $\left[-\mathrm{CH}_{2}-\mathrm{CH}\left(\mathrm{CO}_{2} \mathrm{Na}\right)-\right]_{n}$. 


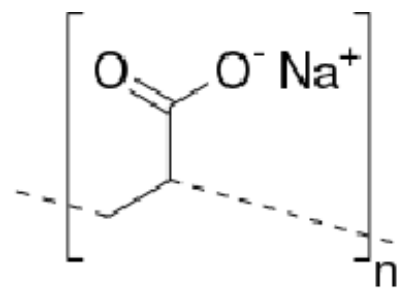

\section{Chemical structure of Sodium polyacrylate}

(B) Cellulose derivatives: Depending upon their type of substitution, cellulose derivatives exhibit different concerts. The most widely used cellulose derivatives as a mucoadhesive polymer are HPC (hydroxyl propyl cellulose), HPMC (hydroxyl propyl methyl cellulose), HEC (hydroxyethyl cellulose) and CMC (carboxymethylcellulose). As compared to other cellulose derivatives CMC shows greater mucoadhesive properties and also high hydrogen-bonding capacity ${ }^{11}$.

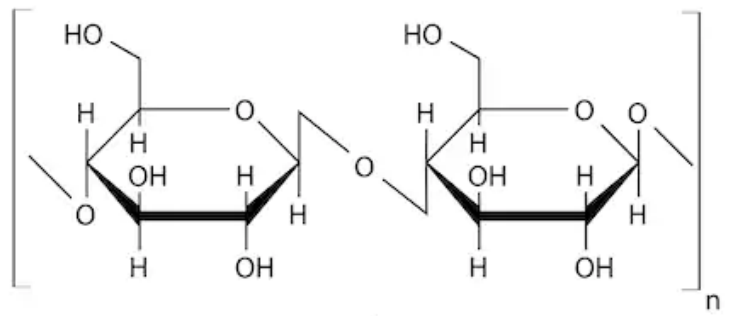

Chemical structure of cellulose

(C) Chitosan: As compared to other cationic polymers, Chitosan is most broadly investigated polymer. Chitosan is a biocompatible, hydrophilic and biodegradable polymer with very low toxicity. Chitosan have moderate swelling and mucoadhesive properties. Used in the formulation of sustained release.

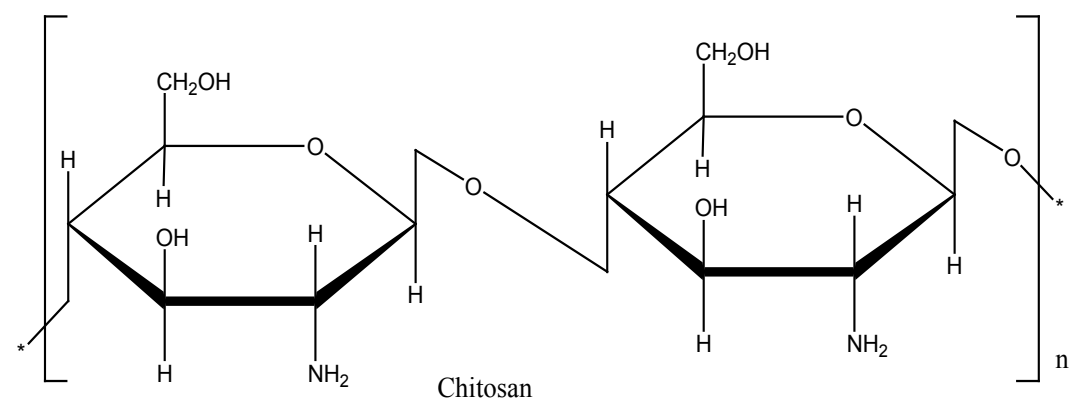

Chemical structure of chitosan 
(D) Alginates: Alginates are naturally occurring polymers and extracted from seaweed, algae, and bacteria. Alginate is a negatively charged polymer and used for the preparation of microparticles because of their good mucoadhesive properties. Both low molecular weight alginate (too stiff) and high molecular weight (more flexible) are used as mucoadhesive polymer ${ }^{12}$.

(E) Pectin: Pectin found in the cell wall of maximum plants and it is a watersoluble and heterogeneous polysaccharide. After mixing pectin and mucin in deionized water, the formation of large aggregates shown the relationship between pectin and mucin with hydrogen bonding.

(F) Polyvinyl Pyrrolidone: PVP is a non-ionic synthetic linear polymer having group (N-vinyl-2-pyrrolidone). PVP is non-toxic and due to this nature, it is used in non-parenteral exposure. PVP is soluble in various solvents (aqueous and organic) and these are chemically inert. It is mostly used in many formulations of pharmaceutical due to its mucoadhesive properties. The mucoadhesive properties of $\mathrm{PVP}$ at the $\mathrm{pH}$ of the intestine is 6.6.

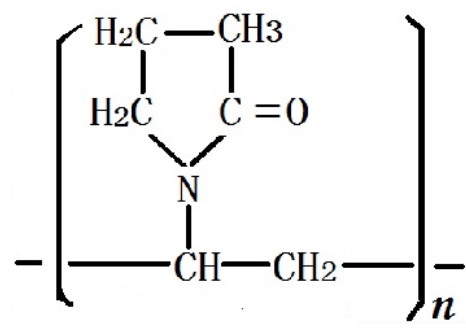

\section{Chemical structure of Polyvinyl Pyrrolidone}

Out of numerous polymers available in nature, Flaxseed mucilage is selected to study the mucoadhesive potential

\section{Flaxseed: Flaxseed mucilage (Linum usitatissimum L.)}

Family: Linaceae

Flaxseed mucilage (FSM) is a mucilage of polysaccharide extracted from fully developed dried seed of linseed or flax plant. Flax seed have two basic varieties: Golden or yellow (also known as golden linseed) and brown flax seed. The mucilage of flax seed contains two different polysaccharide types:- (1) acidic polysaccharides (rich in L- rhamnose, D-galactose) and (2) natural polysaccharides (rich in D-galacturonic acid, L-rhamnogalacturonan) ${ }^{10}$. 

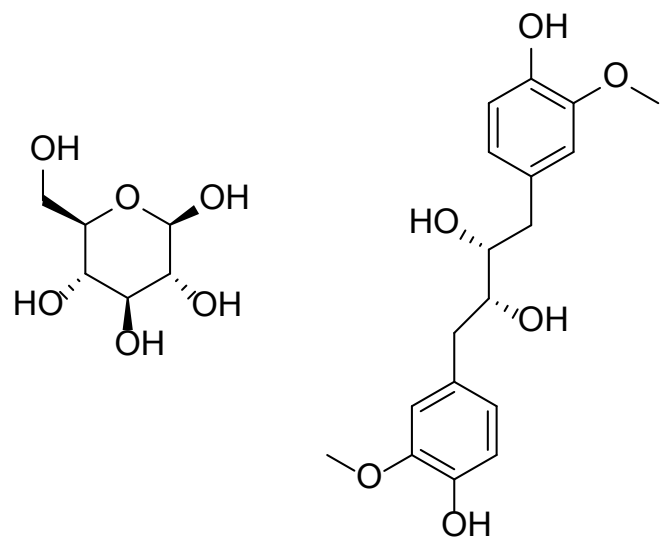

\section{Structure of chemical constituent's flaxseed mucilage}

Flaxseed is well recognized for the content of chemical compounds with specific biological activity and functional properties: polyunsaturated fatty acids (PUFA), omega-3 family, lignans, soluble dietary fibers and carbohydrates ${ }^{13}$. The mucilaginous cells are present on the outer surface of the seed. The seed coat present on the outer surface of the flaxseed protect the flaxseed from mechanical damage and UV radiations ${ }^{3}$.

The seed coat of flax seed have the following roles:

- Providing resistance against rain and wind

- Promoting water uptake

- Facilitating seed disposal by sticking to animals.

Flaxseed is rich in dietary fibers. Flax seed have many biologically active compounds such as linoleic acid, linoleic acid, lignans, polysaccharides, alkaloids, and cyanogenic glycosides. The production of flaxseed is for the production of oil, fiber, and food ${ }^{13}$.

\section{Properties}

It displays a great thickness in liquid or aqueous media and embraces a virtuous water-holding and swelling capability. Moreover, it possesses exclusive nutritive significance as a dietary fiber and gelling agent. Nowadays, flax seed have been used also in pharmaceuticals.

Applications of flax seed

1. Pharmaceutical applications of flax seed

(a) Flaxseed mucilage and gums used information on mucoadhesive tablets be- 
cause of their mucoadhesive nature. It is used as a disintegrant in tablets.

(b) Used as an emulsifying and suspending agent to stabilize the emulsion formulations.

(c) Used as sustaining material in dosage form

(d) Used as coating material

(e) Used as gelling agent ${ }^{15}$

2. Therapeutically applications of flax seed

(a) Used in cardiovascular disease (whole ground flaxseed, fiber, oil, and powder.

(b) Used for rheumatoid arthritis and inflammation of joints (flaxseed oil)

(c) Useful in hyper-cholesterolemia (flax seed powder and flaxseed oil)

(d) Used in the treatment of diabetes

(e) Useful to treat obesity

(f) Used in hypertension

(g) Used to treat tumor or cancer

(h) Used in kidney disease ${ }^{16}$

3. Dietary application of flaxseed: Flaxseed meal is gluten-free with a satisfying nutty (nuts like) flavor. The protein content also found in flax seed meals combined with binding properties of soluble fiber.

Flax seed works as a dietary supplements

(a) Fiber supplementation (bulk laxative with a demulcent action)

(b) Used as a component of protein powder blends.

(c) Many food ingredients (breads and other baked goods) are gluten-free products which are made by using flax seed.

(d) Healthy functional snack food (high protein energy bars)

(e) Oil of flax seed maintain the level of omega- 3 and omega- 6 in the body

(f) Useful in the treatment of constipation and helps to maintain the overall bowel health.

Muco adhesion:

The term muco adhesion refers to any bond made between any two biological surfaces or a bond between a synthetic surface and mucosal surface (biological surface) and the constituents which are proficient for interaction with biological measurable and adhering them together for extended periods are known as mucoadhesive/ bioadhesives.

In bio adhesive drug delivery, the word bio adhesion is used to define the connection between polymers (synthetic or natural) and gastro-intestinal mucosa 
or any soft tissue of the body. In cases when the bond is designed with the mucosa, the term mucoadhesion may be used synonymously with bio adhesion ${ }^{17}$

\section{MECHANISM OF MUCOADHESION ${ }^{17,18}$}

As stated, mucoadhesion is the connection of the drug and a suitable carrier to the mucous membrane. Mucoadhesion is a complex phenomenon which include:

STEP 1. Swelling and Wetting of polymer (contact stage)

STEP 2. Interpretation between the mucosal membrane and chain of polymers STEP 3. Establishment of connections between the tangled chains (both known as consolidation stages). Figure 1. Shows the mechanism of mucoadhesion

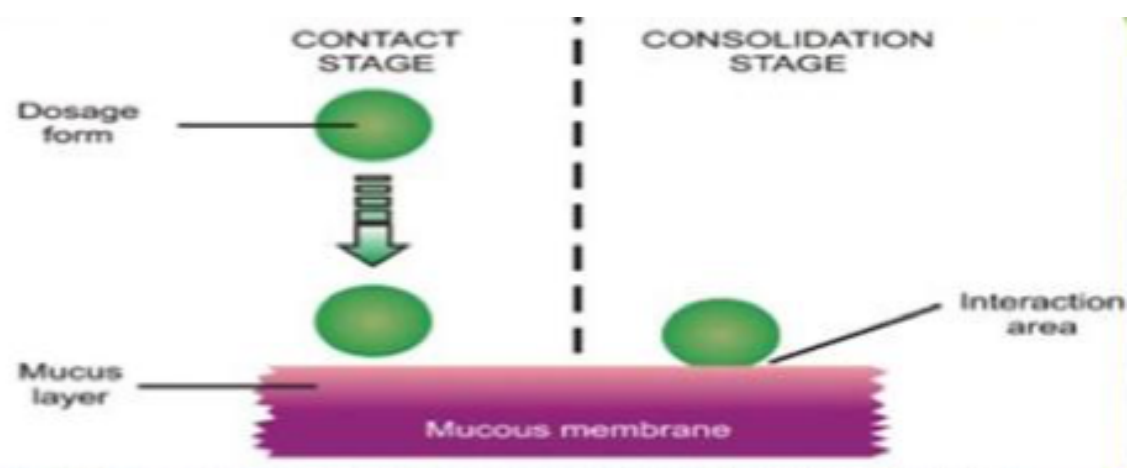

Figure 1. Mechanism of mucoadhesion

Theories of mucoadhesion ${ }^{18,19}$

1. Wettability theory

This theory is useful to low viscosity or liquid mucoadhesive organizations. Wettability theory defines the capability of a mucoadhesive polymer to the extent on biological membrane consequently it provides an interpretation of the "spreadability" of active drug delivery system. The process of wetting theory describes the energy essential to stabilize the surface rigidity at the interface amongst the two surfaces permitting for a virtuous mucoadhesive distribution and contact of the biological substrate. Figure 2. Shows the interaction angle between the device and the mucous membrane of mucoadhesion. 


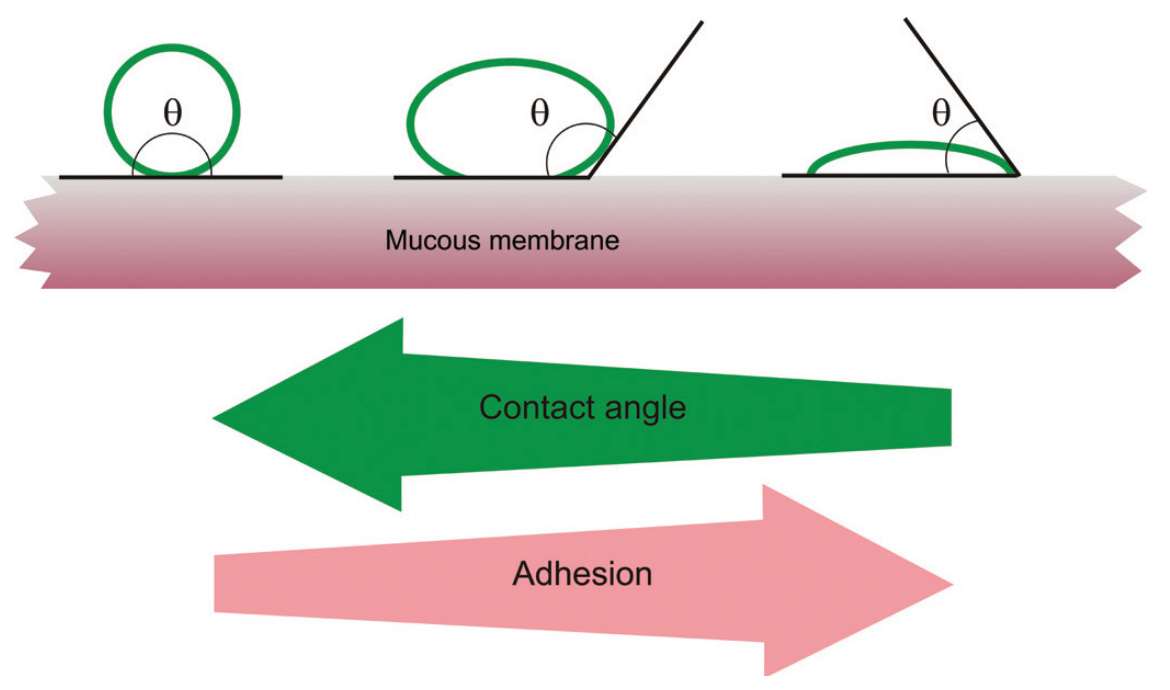

Figure 2. Contact angle between device and mucous membrane

\section{Adsorption theory ${ }^{20}$ :}

Based on the adsorption theory, van der Waals' and hydrogen bond forces established for adhesive interactions. The mucoadhesive substance adhere because of superficial forces performing amongst the molecules of two surfaces, after preliminary contact angle between inter exteriors. According to the concept of chemisorption, collaboration through the interface follows as a consequence of compact covalent bonding.

\section{Fracture theory}

According to this concept, the strength which is required to discrete both the surfaces from each other is connected to the adhesive connections between the systems. This "fracture theory" transmits the strength for polymer impartiality from the mucus to the strength of their adhesive connection (Figure 3). 


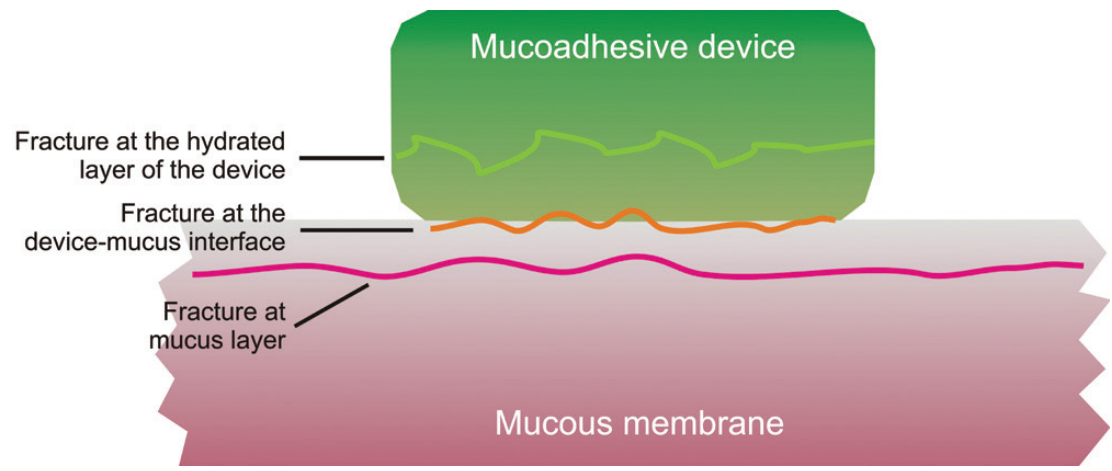

Figure 3. Picture of Secondary interaction resulting from inter-diffusion of polymer chain on bioadhesive device and bioadhesive membrane

\section{Electronic theory}

Each surfaces have their particular electronic structure and structural properties. This system is established on electronic modifications in arrangement or structures. It explains that bonding arises due to electron transmission between the arrangement of polymers and the mucous membrane epithelium. This results, double layer formed by electronic charges formed at mucoadhesive system interface and mucus. This is responsible for the development of attractive force between the two exteriors through the electronic double layer (Figure 4).

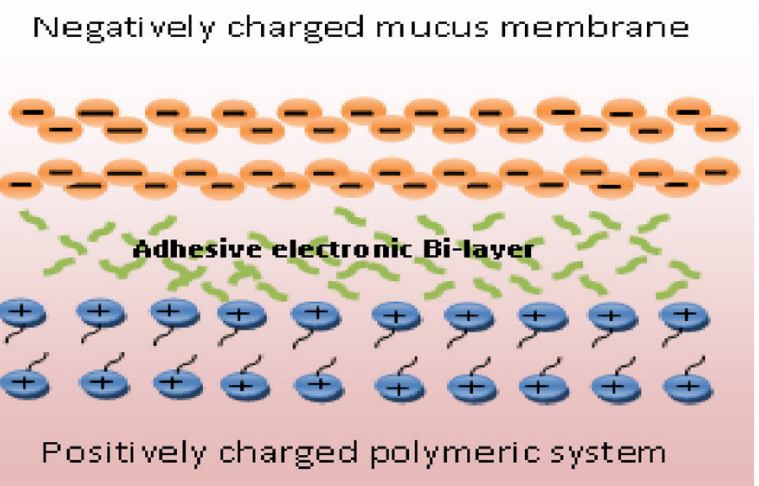

Figure 4. Electronic theory of mucoadhesion. 


\section{Diffusion theory}

This theory explains the time-dependent mucoadhesive polymer chain diffusion into the glycoprotein chain network of the mucus stratum, shows in figure 5 . This is a two-way diffusion method through permeation amount being based upon the diffusion coefficients of mutually relating polymers. While there are several factors elaborated in such procedures, the basic properties that considerably affect this diffusion are cross-linking density, molecular weight, and chain flexibility extension capacity of both networks and temperature (as major factor).

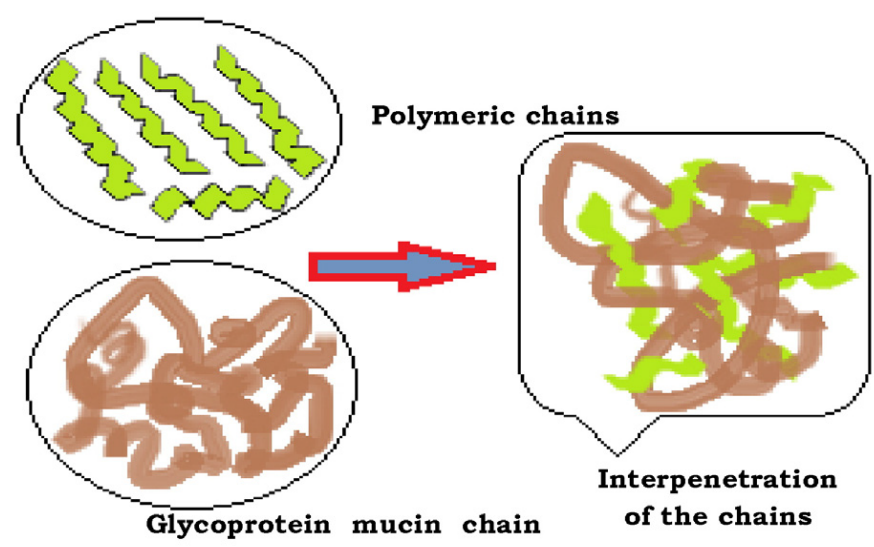

Figure 5. Picture of diffusion interlock of polymeric chains

\section{Factors affecting mucoadhesion process}

The mucoadhesion of the drug carrier system to the mucous membrane depends on the below-mentioned factors ${ }^{18}$. Different types of factors which affect the mucoadhesion process as following

- Concentration of polymer used

- Molecular weight of polymers

- Swelling factors \& stereochemistry of polymer

- Mucin turnover rate

- Flexibility of polymers chain

- Polymer - substrate interaction $\mathrm{pH}^{6,21}$ 


\section{Mucoadhesive drug delivery system}

The use of MDDS is increased day by day because it can increase drug accretion on the mucosa by prolong the dwelling time. Hydrophobic interaction plays a vital role in case of mucoadhesion. In accumulation to electrostatic bonding and hydrophobic interaction, it has been shown that mucoadhesion can also occur through physical interlocking of polymer with mucosal membrane. The mucoadhesive formulations have been prepared as a medication to enhance the contact time of dosage form with the mucosal layer of various routes for drug delivery in body: Oral, Buccal, Oral, Nasal, Rectal, ocular, Sublingual routes ${ }^{22,23}$. For delivery of drug to the oral mucosa observe various areas:

For delivered the mucoadhesive drug by oral route following locations are used:-Oral cavities (lips, tongue, cheek or buccal, soft palate, hard palate and floor of the mouth ${ }^{24}$.

Oral mucosa is a useful term to identify the lining of the oral cavity includes buccal, sublingual, gingival, palatal and labial mucosa.

Importance of mucoadhesive drug delivery ${ }^{25}$

1. It increase the dwelling time of the dosage time at the absorption site.

2. It improves absorption and the therapeutic ability of the drug due to increase the residence time.

3. Exceptional availability ${ }^{21,26}$.

4. Better blood flow rates due to fast blood supply.

5. First, pass metabolism avoidance increase drug bioavailability.

6. Due to the acidic environment in the GIT drug is protected from degradation.

7. Enhanced patient Obedience: faster onset of action is achieved due to mucosal surface and easy to administer.

Limitation of mucoadhesives drug delivery system:

1. Long-time fixation at the site of application ${ }^{26}$.

2. A new perspective is enzyme inhibition and penetration enhancement.

3. Drinking and eating are constrained at the time of mucoadhesive drug delivery.

4. Possibility of swallowing of formulation by patient.

5. Dosage form should not be disseminated after located at the site of absorption. 


\section{Mucoadhesive formulation:}

(A) Tablet

Tablets are solid dosage form and small in size and use mucoadhesive coating. The coating on tablets used for mucoadhesive formulations for adherence of tablet to mucosa for both targeted local and systemic administration. Tablet have less patient compliance when given orally as compared to other route ${ }^{27}$.

(B) Patches

Patches have three separate layers:

(1) Outer impermeable layer

(2) Reservoir layer (middle layer)

(3) Inner layer

Outer layer of patches control the direction of release of drug from the patches at the contact site and middle layer act as reservoir layer and hold the drug and help to provide specified amount of dose at the application site. The final inner layer allows the patch to adhere to the specified mucosa.

(C) Gels

Gels are liquid or semisolid dosage form. When a solid form of any formulation affects the comfort level of the patient then gels are used for treatment. Mucoadhesive formulations increase the viscosity of gels after applications so the drug in the gel form of drug effectively administer at the local sites and also maintaining the comfort of the patient.

(D) Solution

The solution form of mucoadhesive formulation is used when the drug is administered in the eye and nasal cavity. In mucoadhesive formulation, polymers which are mucoadhesive used to improve resistance of mucosal surface. The mucoadhesive system when used for eye then it called situ gelling system.

\section{Advancement in mucoadhesive drug delivery system ${ }^{26}$}

- Liposomes (used to convey vaccine drug, enzyme to cells and organs)

- Nanoparticles (used to improve ocular bioavailability and play a superior role to improve targeting at the site)

- Microsphere

- Nano suspension

- Mucosal vaccination 


\section{Modification of polysaccharides}

Various approaches are used for chemical modifications of these natural polysaccharides are graft polymerization, etherification and cross-linking to overcome the problems of low microbiological stability, disagreeable odor, and uncontrolled hydration limit. Grafting by using vinyl monomers is a very useful application now a day to develop a new and better modified polysaccharides.

\section{(A) Chemical modification}

This technique of modification, can adapt the structure of polysaccharides by announcing substituent groups, and thus reinforce their parent bioactivities as well as generated new useful bioactivities. Some of the chemical modification techniques are:-

(a) Sulfation

This method is used for sulfation of neutral polysaccharides and sulfated polysaccharides.

(b) Carboxy methylation

Cellulose, pachymaran, and scleroglucan have poor water solubility so they can't induce bioactivities. This method is used to raise their biochemical events to stimulate water solubility ${ }^{28}$.

(c) Phosphorylation

Glucose, Fructose, and some other monosaccharides which have no natural bioactivities, can be stimulated after phosphorylated modification.

(d) Acetylation

This method of modification used for the treatment of branches in polysaccharide molecules, which results in improvement in the solubility of polysaccharides.

(e) Alkylation

This method is frequently useful to modify chitosan to decrease their viscosity and improve solubility. Some polysaccharides need to modify by a methyl or ethyl group before analyzed the gas chromatograph is analyzed.

(f) Graft co-polymerization

The graft co-polymerization method involves a long sequence of one or more branches to another polysaccharide. The free radicals sites will create on the performed polymer with the help of external agents ${ }^{2}$.

(g) Etherification 
This method of chemical modification of polysaccharides involves the reaction of an alcohol (Saccharide alcohol) with alkylating agents (carboxylic acid used as alkylating agent).

\section{(h) Oxidation}

Polysaccharides may be oxidized in different ways to produce the structure of different types of polysaccharides. Chemical and biological modification have both seen used for oxidation of primary alcohol of polysaccharides. In oxidation reaction, chemical oxidation of primary alcohol to carboxylic acid and enzymatic oxidation of primary alcohol to aldehyde ${ }^{2}$.

(i) Selenization

Selenium is an essential trace element for the human body and also an important component of glutathione peroxidase and superoxide dismutase. The combination of selenium and polysaccharide into an organic compound can promote the functions of new organic compounds.

\section{(j) Acid/alkali degradation}

This is a traditional method for Production of modified polysaccharides through acid/alkali degradation. To be specific, glycosidic bonds of polysaccharides are first cleaved by acidic/ alkaline solution, thus degradation of polysaccharides into lower molecular weight fragments with high solubility and improved biological activity, etc.

(B) Physical modification

This method is used to shorten the parent polysaccharide strength to develop lower molecular weight fragments. This technique is to confirm the maintenance of the basic arrangement of polysaccharides and the only reason for some conformational variations. The generally used techniques are microwave exposure, ultrasonic disruption, radiation-induced reaction, etc.

(C) Biological modifications

The biological modification of polysaccharides mostly denotes to enzyme modification, which is catalysis with enzymes results degradation of polysaccharides. When biological modification is associated with chemical modification then biological modification shows benefits of great efficiency, high specificity, and low toxic effects. The main aim of biological modification is to destroy the strength of polysaccharide before decreasing its viscosity and its molecular weight. 


\section{Grafted Polysaccharides}

Polysaccharides such as vinyl monomers, Cellulose, gelatin, Starch, Eudragit, Chitosan, Carbopol, Agarose, and Acacia, etc. have been expansively graft modified to achieve macromolecular constituents better to their parent polysaccharides. The graft modified polysaccharide demonstrating better resistance to heat, antibacterial activity, greater oil/water repellant qualities, higher mechanical strength ${ }^{29}$.

In natural form, polysaccharides have reduced shelf life because of their vulnerability to biodegradation but impartially unaffected to degradation below shear and perform as convenient flocculants and synthetic polymers suffer from poor shear dwelling properties but they can be easily tailored. By grafting technique, the synthetic polymers with any natural polysaccharides can be converted into identical customizable matrices with mixture properties appropriate for different uses. Chemical grafting is a useful and most effective method to obtain new material or polysaccharide by increasing the compatibility between natural and synthetic polymers and the new polysaccharide has the most useful and preferred hybrid characteristics. Grafted polysaccharide includes the connection of polymer chains, usually a monomer, to the strengthen polymer ${ }^{30}$.

\section{Grafting Techniques}

The grafting technique is a multipurpose implementation for the copolymer production that may attain innovative property, a new and different arrangement of their parent molecule. Grafting technique carried out with the help of free radicals such as chemical inventors, U.V. Rays in the occurrence of photosensitizers, gamma rays, and microwave radiations. Grafting copolymerization is a new technique for the hybridization of natural polymer with any synthetic polymer for improvement in the functionality of natural polymer and modify a new and better polysaccharide. The most preferred process of graft copolymer production is the use of microwave radiation to produce the free radical sites on the strength polymer ${ }^{29,30}$.

\section{The methods used for graft copolymerization has been classified into various types:}

(1) Grafting initiated by free radicals (chemical method) ${ }^{31}$

(2) Grafting by enzymes (biological method)

(3) Grafting induced by plasma-radiation

(4) Radiation grafting (physical method:microwave radiation-induced grafting) 
(5) Photochemical grafting

(6) Grafting by photochemical reactions

(7) Ionic grafting

(8) Grafting by chemical-free radical initiators etc.

The use of MDDS is increased day by day because it can increase drug accretion on the mucosa by prolong the dwelling time. Hydrophobic interaction plays a vital role in case of mucoadhesion. In accumulation to electrostatic bonding and hydrophobic interaction, it has been shown that mucoadhesion can also occur through physical interlocking of polymer with mucosal membrane. The mucoadhesive formulations have been prepared as a medication to enhance the contact time of dosage form with the mucosal layer of various routes for drug delivery in body- Oral, Buccal, Oral, Nasal, Rectal, ocular, Sublingual routes.

\section{CONFLICT OF INTEREST}

The authors report no conflicts of interest. 


\section{REFERENCES}

1. Akin, A.; \& Isiklan, N. Microwave assisted synthesis and characterization of sodium alginateg-poly (N,N“- Dimethyl acrylamide). Int. J. Biol. Macromol., 2016, (82) 530-540.

2. Bhosale, R.R.; Osmani R.A.; \& Moin A. Review on natural polysaccharide based particulate drug delivery systems: An inimitable tactic in novel drug delivery systems. Int. J. Curr. Pharm. Rev. Res., 2014, 5:138-155.

3. Tomada, M.; Shimada, K.; Satio, Y.; \& Sugi, M. Plant mucilages: Isolation and structural features of Okra mucilage, from the immature fruits of Abelmoschus esculentus . C h e m . Pharm. Bull., 1980, 28(10):2933-40.

4. Bemiller, J.N.; \& Whistler, R.L. Industrial gums: Polysaccharides and their derivatives. Academic Press, 2012.

5. Kulkarni, R.V.; Imamdar, S.Z.; Das, K.K.; \& Biradar, M.S. Polysaccharides- based stimuli sensitive graft co-polymer for drug delivery. Advanced drug delivery review. Woodhead publishing, 2010, 155-171.

6. Boddupalli, B.M.; Mohammed, Z.N.K.; Nath, R.K.; \& Banji, D. Mucoadhesive drug delivery system: A review. J. Adv. Pharm. Technol. Res., 2010, Vol 1 (4): 381-387.

7. Nagaveni, P.; Sekhar, C. K.; \& Reddy, J . Design and in-vitro evaluation of mucoadhesive drug delivery of nateglinide. Int. J. Pharm. Sci. Res., 2017, 8(4): 676-684.

8. Morales, J.O.; \& McConville, J.T. Manufacture and characterization of mucoadhesion buccal films. Eur. J. Pharm. Biopharm., 2011, 77(2). 187-199

9. Singh, J.; \& Deep, P. A Review Article on Mucoadhesive Buccal Drug Delivery System.

Int. J. Pharm. Sci. Res., 2013, 4(3):916.

10. Russo, E.; Selmin, F.; Baldessari, S.; Caviglioli ,G.; Ciluzo, F.; Minghetti, P.; \& Parodhi, B. A focus on mucoadhesive polymers and their applications in buccal dosage forms. J. Drug Delivery Sci. Technol., 2016, 32:113-125

11. Gopinath, V.; Saravanan, S.; Al-Malekin, A.R.; Ramesh, M.; \& Vedivelu, J. Review of natural polysaccharides for drug delivery application. Biomed. Pharmacother., 2018, (107), 96-108.

12. Barclay, T.G.; Day, C.M.; Petrovsky, N.; \& Garg, S. Review of polysaccharide particle-based functional drug delivery. Carbohydr. Polym., 2019, (221) 94-112.

13. Shim, Y.Y.; Gui, B.; Arnison, P.G.; Wang, Y.; \& Reaney, M.J. Flaxseed (Linum usitatissimum L.) bioactive compounds and peptide nomenclature: A review. Trends in fo Trends Food Sci Technol, 2014, 38(1):5-20.

14. Rubilar, M.; Gutierrez, C.; Verdugo, M.; Shene, C.; \& Sineiro, J. Flaxseed as a source of functional ingredients. Soil Sci. Plant Nutr., 2010, 10(3), 373-377.

15. Prajapati, V.D.; Jani, G.K.; Moradiya, N.G.; \& Randeria, N.P. Pharmaceutical applications of various natural gums, mucilages and their modified forms. Carbohydr. Polym., 2013, 92(2):1685-99.

16. Goyal, A.; Sihag, M.K.; Patel, A.; \& Tanwar, B. Therapeutic potential of flax seed. In therapeutic probiotic and unconventional foods. Academic press, 2018, 256-267.

17. Tangri, P.; \& Madhav, N.V.S. Oral mucoadhesive drug delivery system. Int J Biopharmaceutics, 2011, 2(1): 36-46.

18. Mansuri, S.; Kesharwani, P.; Keerti, J.; Rakesh, K.T., \& Jain. Mucoadhesion : A promising approach in drug delivery system. React. Funct. Polym., 2016, 100: 152-173. 
19. Carvalho, C.F.; Braschi, L.M.; Evangelista, R.C.; Gremiao, M.F.D. Mucoadhesive drug delivery system. Braz. J. Pharm. Sci.2010, vol. 46 (1), 1-17.

20. Ahuja, M.; Singh, S.; \& Kumar, A. Evaluation of carboxymethyl gellan gum as a mucoadhesive. Int. J. Biol. Macromol., 2013, 53:114-121.

21. Salamat-Miller, N.; Chittchang, M.; \& Johnston, T.P. The use of mucoadhesive polymers in buccal drug delivery. Adv. Drug Delivery Rev., 2005, 57(11):1666-91.

22. Ahuja, A.; Khar, R.K.; \& Ali, J. Muco adhesive drug delivery system. Drug Dev. Ind. Pharm., 1997, 23(5) 489-515.

23. Pardeshi, C.V.; Rajput, P.V.; Belgamwar, V.S.; \& Tekade, A.R. Formulation, optimization and evaluation of spray-dried mucoadhesive microspheres as intranasal carriers for Valsartan. J. Microencapsulation, 2012, 29(2):103-14.

24. Silva, B.M.A.; Borges, A.F; Silva, C.; \& Simoes, S. Muco adhesive oral films: The potential for unmet needs. Int. J. Pharm.,2015, 494 (1)537-551.

25. Kaur, A.; \& Kaur, G. Mucoadhesive buccal patches based on interpolymer complexes of chitosan-pectin for delivery of carvedilol. Saudi Pharm. J., 2012, 20(1):21-7.

26. Babade, N.N.; Atran, S.C.; Wankhade, V.P.; Pande, S.D.; \& Tabar, K.K. A review on buccal drug delivery system. Int. J. Pharm. Pharm. Sci., 2013, 3(1): 35-40.

27. Russo, E.; Selmin, F.; Baldessari, S.; Caviglioli, G.; Ciluzo, F.; Minghetti, P.; \& Parodhi, B. A focus on mucoadhesive polymers and their applications in buccal dosage forms. J. Drug Delivery Sci. Technol., 2016, 32:113-125.

28. Liu, J.; Shen, J.; Shim, Y.; Reaney, M.J.T. Characterization and solution rheology of flax seed gum carboxy methylation derivatives. Int. J. Food Sci. Technol., 2016, 51(2), 530-541.

29. Singh, V.; Kumar, P.; \& Sanghi, R. Use of microwave irradiation in the grafting modification of the polysaccharides-A review. Prog. Polym. Sci., 2012, 37(2): 340-364.

30. Mishra, S.; Mukul, A.; Sen, G.; \& Jha, U. Microwave assisted synthesis of polyacrylamide grafted starch and its applicability as flocculant for water treatment. Int. J. Biol. Macromol., 2011, 48(1) 106-111.

31. Setia, A. Application of graft copolymerization: A revolutionary approach. Biopolymer grafting, 2018, 1-44. 